www.nature.com/ejhg

\title{
Single nucleotide polymorphisms in the transcobalamin gene: relationship with transcobalamin concentrations and risk for neural tube defects
}

\author{
Lydia A Afman ${ }^{1}$, Karin JA Lievers ${ }^{1}$, Nathalie MJ van der Put ${ }^{1,2}$, Frans JM Trijbels ${ }^{1}$ and \\ Henk J Blom*,1 \\ ${ }^{1}$ University Medical Center Nijmegen, Department of Pediatrics, Laboratory of Pediatrics and Neurology, P.O. Box \\ 9101, 6500 HB Nijmegen, The Netherlands
}

\begin{abstract}
Homocysteine levels are elevated in mothers of neural tube defect (NTD) children, which may be due to a disturbed folate or vitamin B12 metabolism. Vitamin B12 is transported to the tissues by transcobalamin (TC). We previously showed that a low holo-TC/total-TC ratio is a risk factor for NTD, possibly due to an impaired binding of vitamin B12 to TC. The coding region of the TC gene of 12 individuals was analysed for genetic variations responsible for a disturbed vitamin B12 binding. The influence of the genetic variations observed on total-TC, holo-TC, holo-TC/total-TC, erythrocyte vitamin B12, plasma homocysteine concentrations and risk for NTD was explored in $\mathbf{4 2}$ mothers of a child with NTD and in 73 female controls. Direct sequencing analyses revealed five single nucleotide polymorphisms (SNPs). Three SNPs affected total-TC concentrations, whereas two SNPs seem to affect the binding of vitamin B12. None of the genotypes defined by the SNPs had a significant effect on homocysteine levels, or was associated with an increased NTD risk. Among the five SNPs observed only P259R could partly explain the reduced proportion of vitamin $\mathrm{B} 12$ bound to $\mathrm{TC}$, which has been associated with an increased risk for having a child with NTD. Some of the variants studied affected total-TC and holo-TC/total-TC ratio but a larger study population is required to elucidate whether these SNPs influence delivery of vitamin B12 to the tissue, influence homocysteine levels and whether they are associated with an increased NTD risk. European Journal of Human Genetics (2002) 10, 433-438. doi:10.1038/sj.ejhg.5200830
\end{abstract}

Keywords: transcobalamin; vitamin B12; homocysteine; neural tube defects

\section{Introduction}

In man, the neural tube closes during the fourth week of gestation and failure of closure results in a neural tube defect (NTD), among which anencephaly and spina bifida are the most common. Periconceptional

*Correspondence: Dr HJ Blom, UMC Nijmegen, Laboratory of Pediatrics \& Neurology, P.O. Box 9101, 6500 HB Nijmegen, The Netherlands.

Tel: +31 24 3613469; Fax: +31 24 3618900; E-mail: H.Blom@ckslkn.azn.nl ${ }^{2}$ Current address: Unilever Research Vlaardingen, Olivier van Noortlaan 120, 3133 AT Vlaardingen, The Netherlands

Received 11 February 2002; revised 16 April 2002; accepted 26 April 2002 folic acid supplementation reduces the risk of NTD with $50-70 \% .^{1,2}$ So far, the mechanism behind this preventive effect of folic acid supplementation on the occurrence and recurrence of NTD is poorly understood. Besides its involvement in the synthesis of purines and thymidine, folate is also used in the remethylation of homocysteine to methionine. A part of the protective effect of folic acid can be explained by the metabolic effects of the $677 \mathrm{C}>\mathrm{T}$ variation in the methylenetetrahydrofolate reductase (MTHFR) gene. This common variant is the first identified genetic risk factor for 
NTD, which in homozygous state is associated with increased homocysteine levels. ${ }^{3}$ Several studies found that homocysteine levels were elevated in mothers of children with NTD. ${ }^{4-6}$

Homocysteine is remethylated to methionine by methionine synthase (MS) which catalyses the transfer of the methyl group of 5-methyltetrahydrofolate (5-methyl-THF), via vitamin B12, to homocysteine. Several investigators examined a possible association between vitamin B12 concentrations in blood or amniotic fluid and the risk for NTD but with ambiguous outcome. ${ }^{4,7-10}$

In plasma, about $20 \%$ of the vitamin B12 is bound to transcobalamin (TC), and approximately $80 \%$ is bound to haptocorrin (HC). Holo-TC is the proportion of TC that contains vitamin B12, as opposed to apo-TC to which no vitamin $\mathrm{B} 12$ is bound. TC transports the vitamin $\mathrm{B} 12$ to the cell whereas the role of HC is unclear, so far. A reduced cellular vitamin B12 availability may be detected earlier by determination of plasma holo-TC levels than by total plasma vitamin B12 levels, which is the sum of holo-TC and holo-HC.

In our recent study, we found low plasma holo-TC levels to be associated with an increased risk for having a child with NTD (OR: 2.9 95\% CI: 0.9-9.2) whereas, no such association was observed with total plasma vitamin B12 levels. ${ }^{11}$ Moreover, we found that a low percentage of vitamin B12 bound to TC (holo-TC/total-TC) is associated with an increased risk for NTD (OR: 5.0 95\% CI: 1.3-19.3). Because total plasma vitamin B12 levels were not decreased in individuals with low holo-TC levels, our results suggested a disturbed binding of vitamin B12 to TC. We hypothesised that genetic variations in the TC gene may explain an impaired TC function.

In the present study we sequenced the entire coding region of the TC gene in eight individuals with a low and in four individuals with a high proportion vitamin B12 bound to TC and examined the exonic sequences for genetic variations. The influence of the genetic variations observed on total-TC, holo-TC, holo-TC/total-TC ratio, erythrocyte B12, plasma homocysteine levels and risk for NTD was assessed in 46 mothers of NTD children and 73 female controls.

\section{Materials and Methods \\ Study population}

The study population has been described before ${ }^{11}$ and consisted of 46 mothers of patients with non-syndromic NTD (mean age $38 \pm 4$ years), mainly spina bifida, recruited by participation of the Department of Pediatrics of the University Medical Center Nijmegen and of 73 controls (mean age $35 \pm 8$ years). The controls were healthy female employees of our University Medical Center with no family history of NTD. All participants were Dutch Caucasians. DNA of 42 mothers was available with a NTD child and of all 73 controls. The protocol was approved by the local ethics committee and written informed consent was obtained from all participants.

\section{Laboratory methods}

Plasma determination Holo-TC and holo-HC levels were determined in EDTA plasma according to the method described by Benhayoun et al. ${ }^{12}$ Plasma vitamin B12 (holo-TC + holo-HC) was determined using the Dualcount Solid Phase Boil Radioassay (Diagnostic Products Corporation, Los Angeles, CA, USA). TC was removed with Heparin-Conjugated-Sepharose and the remaining holo$\mathrm{HC}$ was also determined by the Dualcount Solid Phase Boil Radioassay. Holo-TC levels were calculated as the difference between holo-HC levels and plasma vitamin B12 levels. Plasma apo-TC and apo-HC levels were determined according to the method described by Nielsen et al. ${ }^{13}$ Homocysteine concentrations were determined in EDTA plasma by HPLC with fluorescence detection. ${ }^{14}$ Erythrocyte vitamin B12 and plasma folate levels were determined by using the Dualcount Solid Phase Boil Radioassay (Diagnostic Products).

Sequencing analyses We selected eight individuals (six mothers of NTD children and two female controls) with low holo-TC levels and a low holo-TC/total-TC ratio. In addition, four subjects (one mother of a NTD child and three female controls) with high holo-TC levels and a high holo-TC/total-TC ratio were selected for DNA analysis of their TC gene. The TC gene consists of nine exons, ${ }^{15}$ which were sequenced using primers that were located in the flanking introns (Table 1). PCRs were carried out in a total volume of $50 \mu \mathrm{l}$, containing $50 \mathrm{ng}$ of the sense and antisense primer (Table 1), $200 \mu \mathrm{M}$ each dNTP, $10 \mathrm{~mm}$ Tris- $\mathrm{HCl}$ buffer ( $\mathrm{pH} 8.3$ ), $1.0 \mathrm{~mm} \mathrm{MgCl}_{2}$ and 0.5 units Taq polymerase (all from Life Technologies, The Netherlands) in a Perkin Elmer 9600 thermocycler (PE Biosystems, The Netherlands). PCR conditions were: initial denaturation at $92^{\circ} \mathrm{C}$ for $2 \mathrm{~min}$, followed by 35 cycles: of $30 \mathrm{~s}$ at $92^{\circ} \mathrm{C}, 30 \mathrm{~s}$ at $47-58^{\circ} \mathrm{C}$, and $30 \mathrm{~s}$ at $72^{\circ} \mathrm{C}$, and a final step at $72^{\circ} \mathrm{C}$ for 7 min. Subsequently, all PCR products were sequenced on both strands using the ABI Prism BigDye Terminator cycle sequencing kit according to the manufacturer's recommendations (PE Biosystems, The Netherlands) on an ABI Prism 377 automated DNA sequencer.

SNP analyses The prevalences of the variants detected by DNA sequencing analysis were analysed by PCR - RFLP and subsequent separation on agarose gels (Table 2). For the screening of S348F in exon 7, an artificial EspI restriction side was created by using the mutagenic sense primer $5^{\prime}$ CCGTACAGACAGTCCGTC-3' that, in combination with the wild type allele, resulted in the EspI recognition site. The prevalences of the previously described P259R and Gln234Arg variants were also determined by ScrFI and MspI restriction enzyme analysis, respectively (Table 2 ). The 
MTHFR $677 \mathrm{C}>\mathrm{T}$ polymorphism was determined as described by Frosst et al. ${ }^{16}$

Prediction of the putative secondary structure changes in the TC protein by each of the SNPs was performed using the Chou-Fasman ${ }^{17}$ and GOR II (Garnier, Osguthorpe, Robson) ${ }^{18}$ algorithms included in the OMIGA version 2.0 software package.

\section{Statistical analyses}

Odds ratios (OR) with 95\% confidence interval (CI) were calculated using logistic regression analyses. Homocysteine levels showed a non-normal distribution; we therefore used logarithmically-transformed homocysteine concentrations in all calculations, and homocysteine concentrations were expressed as geometric means. Differences in mean metabolic variables between the various genotypes were assessed by linear regression analyses. All $P$-values were only adjusted for age, except those concerning homocysteine concentrations which were adjusted for age, plasma folate and the MTHFR $677 \mathrm{C}>\mathrm{T}$ polymorphism. Tests for trend were calculated using linear regression analyses. All tests were performed two-tailed, and statistical significance was accepted at $P<0.05$. All analyses were performed using SPSS software package version 10.0 .

\section{Results}

Direct sequencing analyses of the coding region of the TC gene in 12 selected individuals revealed five sequence variants; each of these, including the previously described Gln234Arg variant was subsequently analysed in the entire study population of 42 NTD mothers and 73 healthy controls (Table 2). The Gln234Arg variant ${ }^{19}$ was not detected in our study groups. Table 3 summarises the genotype distributions in mothers of NTD children and in controls. On the basis of these frequencies we calculated ORs with 95\% confidence interval (CI) as estimates of the relative risk for NTD. No particular genotype was associated with an increased NTD risk.

The $67 \mathrm{~A}>\mathrm{G}$ transition is located in exon 2 and results in an isoleucine by valine replacement at codon 23 (I23V). The allele frequency of the $23 \mathrm{~V}$ allele in controls and in mothers of NTD children was $15 \%$ and $11 \%$, respectively (total study population 13\%). The secondary structure of the TC protein did not change due to this amino acid change, as assessed by both Chou-Fasman and GOR II secondary structure prediction programs.

The $280 \mathrm{G}>\mathrm{A}$ transition is located in exon 3 and mandates a glycine by a serine substitution at codon 94 (G94S). The secondary structure of the mutated polypeptide was identical to its wild type counterpart. This variant was only observed in the heterozygous state and in two mothers of NTD children, resulting in an allele frequency of $2 \%$ (total study population $0.9 \%$ ).

The third variant detected is a $1043 \mathrm{C}>\mathrm{T}$ transition in exon 7, that results in the replacement of serine by phenylalanine at codon 348 (S348F). Again, no change in secondary structure was predicted using the abovementioned algorithms. The allele frequency of the $348 \mathrm{~F}$ allele was $17 \%$ in controls and $14 \%$ in mothers of NTD children (total study population 16\%). The distribution of the genotypes in controls and in mothers of NTD children was similar; however, the homozygous 348FF genotype was not observed among NTD mothers.

In exon 8, we observed an $1196 \mathrm{G}>\mathrm{A}$ transition, that mandates an amino acid change from arginine to glutamine at codon 399 (R399Q). Also, this variant is not expected to change the secondary structure of the TC protein. The R399Q variant was present only in the heterozygous state with an allele frequency of $3 \%$ in controls and $7 \%$ in cases (total study population $4 \%$ ). The prevalence of the 399RQ genotype was higher in mothers of NTD chil-

Table 2 Sequence variations in the TC gene

\begin{tabular}{llll}
\hline & $\begin{array}{l}\text { Nucleotide } \\
\text { change }\end{array}$ & $\begin{array}{l}\text { Amino acid } \\
\text { change }\end{array}$ & RFLP \\
\hline Exon 2 & $67 \mathrm{~A}>\mathrm{G}$ & Ile23Val & + Rsal \\
Exon 3 & $280 \mathrm{G}>\mathrm{A}$ & Gly94Ser & + AlwNI \\
Exon 5 & $701 \mathrm{~A}>\mathrm{G}$ & Gln234Arg & + Mspl \\
Exon 6 & $776 \mathrm{C}>\mathrm{G}$ & Pro259Arg & - ScrF \\
Exon 7 & $1043 \mathrm{C}>\mathrm{T}$ & Ser348Phe & - Espl \\
Exon 8 & $1196 \mathrm{G}>\mathrm{A}$ & Arg399Gln & - BsmAl \\
\hline
\end{tabular}

${ }^{a}$ Restriction fragment length polymorphism; *Artificially created restriction site.

Table 1 Primers used for the molecular genetic analysis of the TC gene

\begin{tabular}{lll}
\hline & Forward primer $\left(5^{\prime} \rightarrow 3^{\prime}\right)$ & Reverse primer $\left(5^{\prime} \rightarrow 3^{\prime}\right)$ \\
\hline Exon 1 & GGA AGC TGC GTC TCT CGG & GAT GTG TTA AAT GCT GAG GTC \\
Exon 2 & GGT GTG TGC TGG GTG GAG & AGT ACC CAA GT TCC CAT CTC \\
Exon 3 & TGG GGT GGG TGC TGT AAG & TGG ATG GTG GGA AAG ACG \\
Exon 4 & GGG CTG AGG CAA TGA AGG & TTC GGA GGA GCC CCA AG \\
Exon 5 & CTC AAG CCC CTG CCT GTC & AGA GAG AGA GGA AGT CAG CCG \\
Exon 6 & TCA GGG GTG GAG TGG TCA G & ATT TTG GCT GCT GTG TCC C \\
Exon 7 & GGG AAG ACA AGA AGA CAA ATA ATC & TGT TCC CCG TCT CTT CTG TG \\
Exon 8 & TTT TGC TGC TGT GGG TGA G & GCT GGG GTG AGT TGA TGA AG \\
Exon 9 & CTG GAA GAT GAG GTT GCG G & AGG GAC ATC AGG AGG GAG G \\
\hline
\end{tabular}

All primers are based on intronic sequences (Genbank Acc. No: AC005006) 
Table 3 Distribution of the SNPs in the TC gene in mothers of NTD children and controls

\begin{tabular}{llllll}
\hline & Genotype & $\begin{array}{l}\text { Mothers } \\
(n=42)\end{array}$ & $\begin{array}{l}\text { Controls } \\
(n=73)\end{array}$ & $\begin{array}{l}\text { Odds } \\
\text { ratios }\end{array}$ & $\begin{array}{l}95 \% \\
C l\end{array}$ \\
\hline lle23Val* & II & $34(81 \%)$ & $53(74 \%)$ & $1^{\text {a }}$ & - \\
& IV & $7(17 \%)$ & $17(24 \%)$ & 0.6 & $0.2-1.7$ \\
Gly94Ser & GV & $1(2 \%)$ & $2(3 \%)$ & 0.8 & $0.1-8.9$ \\
& GG & $40(95 \%)$ & $73(100 \%)$ & - & - \\
& GS & $2(5 \%)$ & 0 & - & - \\
Pro259Arg & SS & 0 & 0 & - & - \\
& PP & $11(26 \%)$ & $22(30 \%)$ & $1^{\text {a }}$ & - \\
& PR & $25(60 \%)$ & $36(49 \%)$ & 1.4 & $0.6-3.4$ \\
Ser340Phe & RR & $6(14 \%)$ & $15(21 \%)$ & 0.8 & $0.2-2.6$ \\
& SS & $30(71 \%)$ & $50(68 \%)$ & $1^{\text {a }}$ & - \\
& SF & $12(29 \%)$ & $21(29 \%)$ & 1.0 & $0.4-2.2$ \\
Arg399GIn & FF & 0 & $2(3 \%)$ & - & - \\
& RR & $36(86 \%)$ & $69(94 \%)$ & $1^{\text {a }}$ & - \\
& RQ & $6(14 \%)$ & $4(6 \%)$ & 2.9 & $0.8-10.8$ \\
& QQ & 0 & 0 & - & - \\
\hline
\end{tabular}

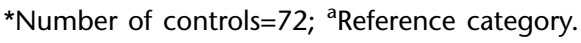

dren although this did not lead to a significantly increased NTD risk (OR: $2.9(0.8-10.8)$ ) (Table 3).

We also encountered the previously described P259R variant, which has an allele frequency of $45 \%$ in controls and $44 \%$ in mothers of NTD children (total study population $45 \%)$. In our study population none of the genotypes defined by this polymorphism was associated with an increased NTD risk. According to the Chou-Fasman algorithm the P259R substitution will change the secondary structure of the protein: a tendency to form helix structures occurred by the substitution of proline at codon 259 by arginine. A change in secondary structure was also predicted by using the GOR II method, which revealed a tendency to form turn structures.

To establish whether the SNPs influence TC function, we investigated its effects on the total-TC, holo-TC, holo-TC/ total-TC ratio, erythrocyte B12 and plasma homocysteine concentrations. Mean concentrations of these parameters were calculated for each of the genotypes defined by the SNPs and are depicted in Figure 1. The most pronounced effect was observed on total-TC concentrations (Figure 1A). The heterozygous 23IV genotype was associated with significantly lower total-TC concentrations compared with the 23II wild type. Those individuals with the homozygous 259RR and heterozygous 259PR genotypes also had lower total-TC concentrations compared with 259PP homozygotes, with the lowest concentration observed in the 259RR peers. Also the R399Q genotypes influenced the total-TC concentration: the 399RQ heterozygotes showed a higher total-TC compared with the 399RR homozygotes.

An effect on holo-TC concentrations was only observed for the P259R variant; homozygous 259RR individuals had a significantly lower holo-TC concentration compared with those with the 259PP genotype (Figure 1B).

The proportion vitamin B12 bound to TC, calculated by the holo-TC/total-TC ratio, was significantly higher in
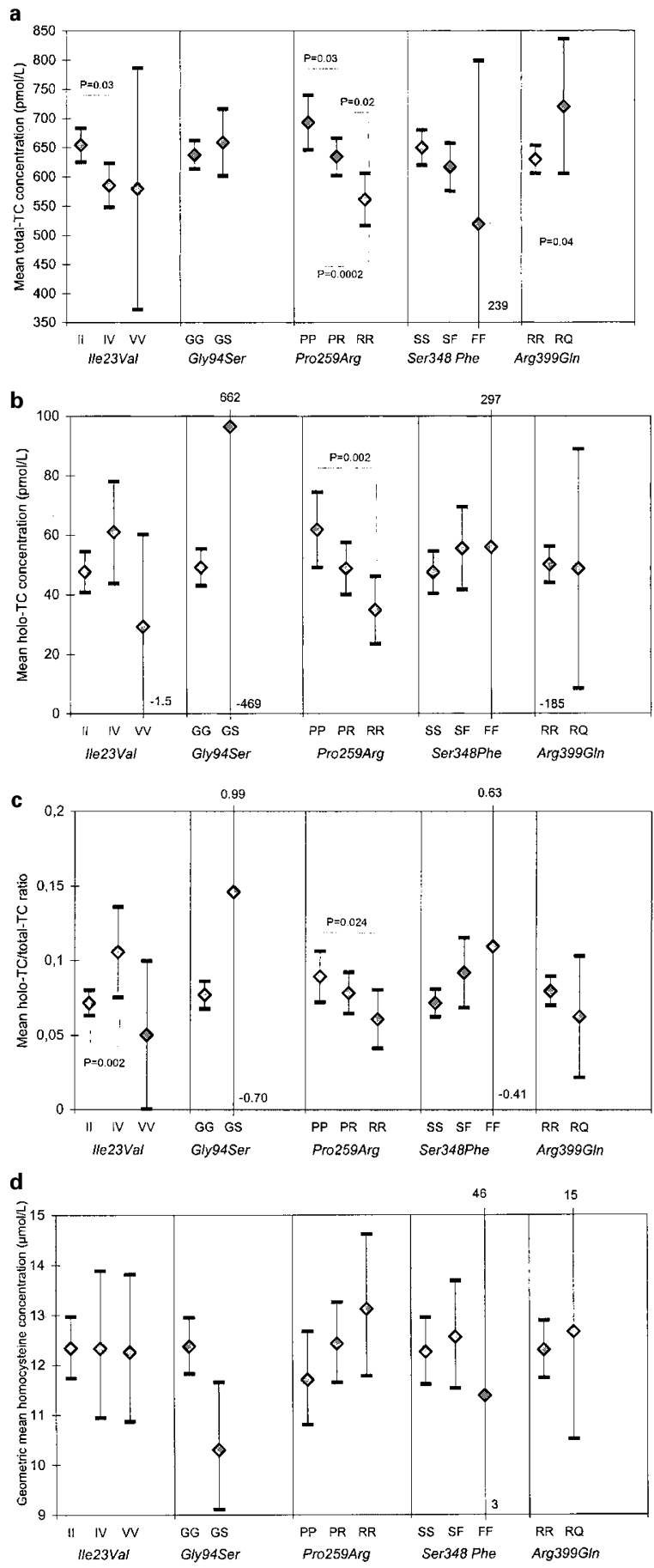

Figure 1 Associations of SNPs in the transcobalamin (TC) gene with mean total-TC (A), holo-TC (B), holo-TC/total-TC ratio (C) and geometric mean homocysteine (D) concentrations. Mean levels are shown as diamonds, $95 \% \mathrm{Cl}$ as thin vertical lines. The values of the $\mathrm{Cl}^{\prime}$ s exceeding the graph are indicated in the figure. $P$-values are only shown when significant differences were observed $(P<0.05)$. All $P$-values were adjusted for age and $P$ values for homocysteine were also adjusted for plasma folate and the MTHFR $677 \mathrm{C}>\mathrm{T}$ polymorphism. 
heterozygous 23IV individuals compared with their wild type 23II peers. The homozygous 259RR genotype was associated with a significantly lower proportion vitamin B12 bound to TC compared with the wild type. The S348F showed a significant trend $(P=0.03)$ in increasing proportion vitamin $\mathrm{B} 12$ bound to $\mathrm{TC}$ with the presence of this SNP (Figure 1C).

We subsequently studied the association between TC genotypes and plasma homocysteine concentrations. None of the TC genotypes was associated significantly with elevated plasma homocysteine concentrations, although an apparent trend was observed for the P259R variant (Figure 1D). In addition, vitamin B12 levels in erythrocytes were not influenced by the TC genotypes (data not shown).

Finally, we calculated mean apo-TC levels for each P259R genotype, to elucidate whether the decrease in total-TC concentration related to the $\mathrm{P} 259 \mathrm{R}$ variant was merely due to a decrease in holo-TC or also due to a decrease in apo-TC. Individuals homozygous for the arginine residue showed lower apo-TC concentrations compared with individuals with the heterozygous 259PR $(P=0.05)$ and wild type 259PP genotypes $(P=0.002)$. In addition, individuals with the heterozygous form of this variant tended to have lower apo-TC concentrations than the wild type individuals $(P=0.07)$. The association between the P259R genotypes and decreased proportion bound vitamin B12 to TC could not be explained by low vitamin B12 levels in blood because holo-HC levels, which represent $80 \%$ of vitamin B12 in plasma, were similar in the three genotype groups (data not shown).

\section{Discussion}

DNA sequencing analyses of the coding region of the TC gene revealed five variants: I23V, G94S, P259R, S348F and the R399Q. Some of the genotypes defined by these variants influenced total-TC protein content in plasma, holo-TC concentrations and the proportion vitamin B12 bound to TC: however, no effect on homocysteine and NTD risk could be detected.

The previously published $\mathrm{P} 259 \mathrm{R}$ variant $^{11,19}$ was associated with a distinct reduction in total-TC protein and apo-TC. Moreover, it was also associated with a pronounced decreased holo-TC concentration and holo-TC/total-TC ratio. A possible explanation for the latter could be a low total vitamin B12 status. However, holo-HC and total vitamin B12 concentrations were not different between the three P259R genotypes. Therefore, the low holo-TC/totalTC ratio may be explained by a reduced binding of vitamin B12 to TC. In spite of this observation, this variant cannot explain the elevated risk for NTD associated with a low proportion vitamin $\mathrm{B} 12$ bound to $\mathrm{TC}$ as reported by us previously, $^{11}$ as the prevalence of this variant was even lower in mothers of NTD children compared with controls. No significant effect of the P259R polymorphism was found on erythrocyte vitamin B12 and plasma homocysteine concentrations, although a tendency towards higher homocysteine levels in homozygous 259RR individuals compared with $259 \mathrm{PP}$ subjects was found $(P=0.07)$. Larger studies are required to elucidate whether this $\mathrm{P} 259 \mathrm{R}$ variant affects plasma homocysteine, intracellular vitamin B12 concentrations and NTD risk.

The effect of the P259R variant on TC levels may be explained by differences in charge of the protein resulting from the substitution of a proline by an arginine, i.e, a neutral by a positively charged one. ${ }^{19}$ According to ChouFasman secondary structure predictions, a tendency to form helix structures was predicted as previously described by $\mathrm{Li}$ et al. ${ }^{19}$ Although the helix structure was not confirmed using the GOR II algorithm, ${ }^{19}$ we found that the latter method predicted a tendency to form turn structures. Both predictions point towards a change in secondary structure. The P259R is not located in one of the six hydrophobic regions, postulated to form the hydrophobic pocket for vitamin B12 binding. ${ }^{20}$ Nevertheless, due to the intra-molecular folding, the P259R substitute may result in a disturbed binding of vitamin B12 to TC.

Our results partly corroborate those of Namour et $a l^{21}$ who also found an association of the P259R variant with decreased apo-TC concentrations in 159 healthy Caucasians. They observed significantly lower homocysteine levels in 259RR homozygotes compared with the heterozygous 259PR individuals. In contrast, our results point towards elevated homocysteine levels in 259RR homozygotes. In both studies the number of individuals included, may not have been sufficient to elucidate whether this SNP is associated with increased homocysteine concentrations.

Heterozygosity for the I23V variant was associated with lower total-TC concentrations, however, it lead to a higher proportion vitamin B12 bound to TC. The I23V variant was previously described by Li et al. ${ }^{15}$ In that study, the coding region of the TC gene was aligned to the coding regions of intrinsic factor (IF) and one of the two haptocorrins, termed 'TCI' in that study. The authors reported that this variant was located in a conserved region of the TC protein; however, both IF and 'TCI' encode a valine instead of an isoleucine at codon 23 , which may indicate that the substitution of isoleucine by valine may even improve vitamin B12 binding. In addition, the I23V SNP was not located in one of the six hydrophobic regions postulated to be involved in vitamin B12 binding.

The R399Q variant is associated with increased total-TC concentrations but does not seem to affect vitamin B12 binding and erythrocyte vitamin B12 or plasma homocysteine concentrations. In addition, the R399Q is not situated in one of the six hydrophobic regions thought to be involved in vitamin B12 binding. More individuals have to be examined to elucidate whether this SNP affects metabolic variables and whether it is associated with an increased risk for NTD. 
The allele frequencies of some of the SNPs studied are very low. This applies to the G94S, for which only two individuals are heterozygous. The homozygous 23VV genotype was found in only three individuals and the homozygous $348 \mathrm{FF}$ was observed in two individuals. The S348F showed a trend towards a higher proportion B12 bound to TC. A consequence of the low prevalences is that this study on potential effects of these SNPs on metabolic variables and on the risk for NTD is lacking power. Examination of these SNPs in larger studies may reveal a potential effect on TC function.

In summary, none of the SNPs found in the TC gene was associated with a significantly increased risk for NTD. The P259R variant, that is expected to change the secondary structure of TC, can only partly explain the reduced proportion vitamin B12 bound to TC, which is associated with an elevated NTD risk. Some of the SNPs seem to affect TC concentrations or function; however, the relatively small number of individuals in our study precludes a conclusion whether these SNPs affect vitamin B12 and plasma homocysteine levels. Therefore, larger studies on these aspects are warranted. In addition, expression of the TC SNPs in an appropriate expression system may provide more information about their possible effects on TC function. Furthermore, in specific situations, some of these TC variants may critically effect homocysteine remethylation and risk for disease for example in individuals with a low B12 status.

The SNPs observed in the coding region of the TC gene can only partly explain the reduced binding of vitamin B12 to TC, related to a fivefold increased risk for NTD. ${ }^{11}$ Possibly, non-coding regions of the TC gene contain variations that influence splicing, affecting TC function. Further research should focus on the non-coding region of the TC gene, using proteomics and RNA technology, to find an explanation for the disturbed binding of vitamin B12 to TC in mothers of NTD children.

\section{Acknowledgements}

We gratefully acknowledge the support of LAJ Kluijtmans and D van Oppenraaij-Emmerzaal. This study was supported by grant no. OO108 from the Prinses Beatrix Fonds, The Netherlands and by grant no. D97.021 of the Netherlands Heart Foundation

\section{References}

1 Czeizel AE, Dudas I: Prevention of the first occurrence of neuraltube defects by periconceptional vitamin supplementation. $N$ Engl J Med 1992; 327: 1832 - 1835.
2 Anonymous: Prevention of neural tube defects: results of the Medical Research Council Vitamin Study. MRC Vitamin Study Research Group. Lancet 1991; 338: 131-137.

3 van der Put NM, Steegers-Theunissen RP, Frosst P et al: Mutated methylenetetrahydrofolate reductase as a risk factor for spina bifida. Lancet 1995; 346: 1070-1071.

4 Steegers-Theunissen RP, Boers GH, Trijbels FJ et al: Maternal hyperhomocysteinemia: a risk factor for neural-tube defects? Metabolism 1994; 43: 1475 - 1480.

5 van der Put NM, Thomas CM, Eskes TK et al: Altered folate and vitamin B12 metabolism in families with spina bifida offspring. QJM 1997; 90: $505-510$.

6 Mills JL, McPartlin JM, Kirke PN et al: Homocysteine metabolism in pregnancies complicated by neural-tube defects. Lancet 1995; 345 : $149-151$.

7 Mills JL, Tuomilehto J, Yu KF et al: Maternal vitamin levels during pregnancies producing infants with neural tube defects. J Pediatr 1992; 120: $863-871$.

8 Kirke PN, Molloy AM, Daly LE, Burke H, Weir DG, Scott JM: Maternal plasma folate and vitamin B12 are independent risk factors for neural tube defects. QJM 1993; 86: 703-708.

9 Steegers-Theunissen RP, Boers GH, Blom HJ et al: Neural tube defects and elevated homocysteine levels in amniotic fluid. Am J Obstet Gynecol 1995; 172: 1436-1441.

10 Steen MT, Boddie AM, Fisher AJ et al: Neural-tube defects are associated with low concentrations of cobalamin (vitamin B12) in amniotic fluid. Prenat Diagn 1998; 18: 545-555.

11 Afman LA, van der Put NM, Thomas CM, Trijbels JM, Blom HJ: Reduced vitamin B12 binding by transcobalamin II increases the risk of neural tube defects. QJM 2001; 94: 159-166.

12 Benhayoun S, Adjalla C, Nicolas JP, Gueant JL, Lambert D: Method for the direct specific measurement of vitamin B12 bound to transcobalamin II in plasma. Acta Haematol 1993; 89: 195 - 199.

13 Nielsen C, Odgaard G: FK 383 DS, a new silica gel for the determination of unsaturated haptocorrin and transcobalamin II in serum. Scand J Clin Lab Invest 1992; 52: 767-769.

14 Te Poele-Pothoff M, van den Berg M, Franken DG et al: Three different methods for the determination of total homocysteine in plasma. Ann Clin Biochem 1995; 32: 218-220.

15 Li N, Seetharam S, Seetharam B: Genomic structure of human transcobalamin II: comparison to human intrinsic factor and transcobalamin I. Biochem Biophys Res Commun 1995; 208: 756 764.

16 Frosst $\mathrm{P}$, Blom HJ, Milos R et al: A candidate genetic risk factor for vascular disease: a common mutation in methylenetetrahydrofolate reductase. Nat Genet 1995; 10: 111-113.

17 Chou PY, Fasman GD: Conformational parameters for amino acids in helical, beta-sheet, and random coil regions calculated from proteins. Biochemistry 1974; 13: 211-222.

18 Garnier J, Osguthorpe DJ, Robson B: Analysis of the accuracy and implications of simple methods for predicting the secondary structure of globular proteins. J Mol Biol 1978; 120: 97-120.

$19 \mathrm{Li} \mathrm{N}$, Sood GK, Seetharam S, Seetharam B: Polymorphism of human transcobalamin II: substitution of proline and/or glutamine residues by arginine. Biochim Biophys Acta 1994; 1219: $515-520$.

20 Li N, Seetharam S, Lindemans J, Alpers DH, Arwert F, Seetharam $B$ : Isolation and sequence analysis of variant forms of human transcobalamin II. Biochim Biophys Acta 1993; 1172: 21 -30.

21 Namour F, Olivier J, Abdelmouttaleb I et al: Transcobalamin codon 259 polymorphism in HT-29 and Caco-2 cells and in Caucasians: relation to transcobalamin and homocysteine concentration in blood. Blood 2001; 97: 1092-1098. 